\title{
Multiwavelength modeling of the Vela pulsar pulses - from Optical light to VHE gamma-rays
}

\section{Bronisław Rudak*}

Nicolaus Copernicus Astronomical Center, Polish Academy of Sciences

E-mail: bronek@ncac.torun.pl

\section{Jarosław Dyks}

Nicolaus Copernicus Astronomical Center, Polish Academy of Sciences

E-mail: jinx@ncac.torun.pl

The pulsed radiation from PSR B0833-45 (Vela) has a phased-averaged spectral energy distribution of an apparently simple structure across a wide energy range. However, in narrow energy bands the pulses reveal astonishing complexity of the directional pattern of the radiation. We present the results of a 3D modeling of the Vela radiation properties in the outer-gap scenario. We show how the synchrotron emission as well as the inverse Compton scattering (ICS) of soft photons by secondary $\mathrm{e}^{ \pm}$-pairs in its magnetic and non-magnetic regimes reproduces qualitatively, and in some cases quantitatively, the observed energy-dependent pulses of Vela. Moreover, we present how ICS of soft synchrotron photons by primary particles can form a pulsed spectral component in the VHE domain. The flux of this component should be of interest to the Cherenkov Telescope Array.

7th Fermi Symposium 2017

15-20 October 2017

Garmisch-Partenkirchen, Germany

\footnotetext{
*Speaker.
} 